\title{
EVERY BANACH IDEAL OF POLYNOMIALS IS COMPATIBLE WITH AN OPERATOR IDEAL
}

\author{
DANIEL CARANDO, VERÓNICA DIMANT, AND SANTIAGO MURO
}

\begin{abstract}
We show that for each Banach ideal of homogeneous polynomials, there exists a (necessarily unique) Banach operator ideal compatible with it. Analogously, we prove that any ideal of $n$-homogeneous polynomials belongs to a coherent sequence of ideals of $k$-homogeneous polynomials.
\end{abstract}

\section{INTRODUCTION}

Most examples of polynomial ideals are inspired in some ideal of operators. This is the case, for example, of the ideals of nuclear, integral, compact, multiple $r$-summing or $r$-dominated polynomials. However, the extension of a linear operator ideal to higher degrees is not always obvious. For example, many extensions of the ideal of absolutely $r$-summing operators have been developed, among them, the absolutely, the multiple and the strongly $r$-summing polynomials and the $r$-dominated polynomials.

The question tackled in this article is whether every ideal of polynomials is an extension of an ideal of operators. This is not a precise question unless we settle what is understood by an "extension of an ideal of operators".

In [5] we defined and studied the concept of a homogeneous polynomial ideal being compatible with an operator ideal. This notion aims to clarify the relationship between an operator ideal $\mathfrak{A}$ and a polynomial ideal defined "in the spirit of $\mathfrak{A}$ ". Compatibility is related with the natural operations of fixing variables and multiplying by linear functionals. We proved in [5, Proposition 1.6] that each ideal of polynomials can be compatible with, at most, one ideal of operators. On the other hand, an operator ideal is always compatible with several different polynomial ideals. In this article we complete these results by showing that a Banach ideal of polynomials is always compatible with a (necessarily unique) ideal of operators.

The concept of coherent sequence of polynomial ideals was also introduced in [5], in the spirit of Nachbin's holomorphy types [13]. It is natural to ask if every ideal of homogeneous polynomials belongs to a coherent sequence. We answer this question affirmatively: for a fixed ideal of $n$-homogeneous polynomials $\mathfrak{A}_{n}$, there exist polynomial ideals

2000 Mathematics Subject Classification. Primary 47H60, 47L20. Secondary 46G25.

Key words and phrases. Polynomial ideals, operator ideals.

Partially supported by CONICET-PIP 11220090100624 and ANPCyT PICT 05 17-33042. The first and third authors were also partially supported by UBACyT Grant X038 and ANPCyT PICT 0600587. 
$\mathfrak{A}_{1}, \ldots, \mathfrak{A}_{n-1}, \mathfrak{A}_{n+1}, \ldots$ such that, together with $\mathfrak{A}_{n}$, form a coherent sequence. That is, a sequence of polynomial ideals of degree $k(k \neq n-1)$ all "associated" to $\mathfrak{A}_{n}$.

Several authors have considered analogous concepts to relate spaces of polynomials of different degrees. This is the case of the ideals of polynomials "closed under differentiation" or "closed for scalar multiplication" introduced in [3], the polynomial property $(B)[2]$ and the already mentioned holomorphy types (see the comments after Definitions 2.1 and 3.1).

Throughout this paper $E, F$ and $G$ will be real or complex Banach spaces (although the proofs and the estimates for compatibility and coherence constants will be given only for the complex case). We denote by $\mathcal{L}(E, F)$ the Banach space of all continuous linear operators from $E$ to $F$ and by $\mathcal{P}^{n}(E, F)$ the Banach space of all continuous $n$-homogeneous polynomials from $E$ to $F$. If $P \in \mathcal{P}^{n}(E, F)$, there exists a unique symmetric $n$-linear mapping $\stackrel{\vee}{P}: \underbrace{E \times \cdots \times E}_{n} \rightarrow F$ such that

$$
P(x)=\stackrel{\vee}{P}(x, \ldots, x) .
$$

We define $P_{a^{k}} \in \mathcal{P}^{n-k}(E, F)$ by

$$
P_{a^{k}}(x)=\stackrel{\vee}{P}\left(a^{k}, x^{n-k}\right) .
$$

For $k=1$, we write $P_{a}$ instead of $P_{a^{1}}$.

A normed ideal of continuous $n$-homogeneous polynomials $[9,10,11]$ is a pair $\left(\mathfrak{A}_{n},\|\cdot\|_{\mathfrak{A}_{n}}\right)$ such that:

(i) $\mathfrak{A}_{n}(E, F)=\mathfrak{A}_{n} \cap \mathcal{P}^{n}(E, F)$ is a linear subspace of $\mathcal{P}^{n}(E, F)$ and $\|\cdot\|_{\mathfrak{A}_{n}(E, F)}$ is a norm on it.

(ii) If $T \in \mathcal{L}\left(E_{1}, E\right), P \in \mathfrak{A}_{n}(E, F)$ and $S \in \mathcal{L}\left(F, F_{1}\right)$, then $S \circ P \circ T \in \mathfrak{A}_{n}\left(E_{1}, F_{1}\right)$ and

$$
\|S \circ P \circ T\|_{\mathfrak{A}_{n}\left(E_{1}, F_{1}\right)} \leq\|S\|\|P\|_{\mathfrak{A}_{n}(E, F)}\|T\|^{n} .
$$

(iii) $z \mapsto z^{n}$ belongs to $\mathfrak{A}_{n}(\mathbb{K}, \mathbb{K})$ and has norm 1 .

\section{Existence of a COMpatible operator IDEAL}

In [5] we defined and studied the concept of a polynomial ideal being compatible with an operator ideal. Let us recall the definition.

Definition 2.1. Let $\mathfrak{A}$ be a normed ideal of linear operators. We say that the normed ideal of n-homogeneous polynomials $\mathfrak{A}_{n}$ is compatible with $\mathfrak{A}$ if there exist positive constants $A$ and $B$ such that for all Banach spaces $E$ and $F$, the following conditions hold:

(i) For each $P \in \mathfrak{A}_{n}(E, F)$ and $a \in E, P_{a^{n-1}}$ belongs to $\mathfrak{A}(E ; F)$ and

$$
\left\|P_{a^{n-1}}\right\|_{\mathfrak{A}(E, F)} \leq A\|P\|_{\mathfrak{A}_{n}(E, F)}\|a\|^{n-1} .
$$


(ii) For each $T \in \mathfrak{A}(E, F)$ and $\gamma \in E^{\prime}, \gamma^{n-1} T$ belongs to $\mathfrak{A}_{n}(E, F)$ and

$$
\left\|\gamma^{n-1} T\right\|_{\mathfrak{A}_{n}(E, F)} \leq B\|\gamma\|^{n-1}\|T\|_{\mathfrak{A}(E, F)} .
$$

It should be noted that property $(i)$ in Definition 2.1 implies the polynomial ideal to be closed under differentiation in the sense of [3].

The examples of ideals of polynomials enumerated in the first sentence of the Introduction are normed, provided that $n \leq r$ for the case of $r$-dominated $n$-homogeneous polynomials, and they are compatible with the respective ideal of operators [5, Section 1]. On the other hand, the ideals of absolutely and strongly $p$-summing polynomials are not compatible with the ideal of absolutely $p$-summing operators, see [5, Example 1.15] and Proposition 2.10 below.

Remark 2.2. Although the definition of compatibility involves constants which relate the norms of the operators and the homogeneous polynomials, a simple application of the closed graph theorem shows that when the ideals are complete those constants automatically exist (see [12] for details). This means that if we can define the operations of fixing variables and multiplying by functionals, then they are bounded, uniformly on the Banach spaces $E, F$.

Even though it is not necessary to obtain the constants $A$ and $B$ to show that two Banach ideals are compatible, in the complex case we will also seek "good" constants (independent of $n$ ) mostly for two reasons: the first one is that they provide a bound for the norm of the derivatives of homogeneous polynomials in different ideals and the second is that this kind of bounds allows us to define holomorphic mappings associated to sequences of ideals [6]. For the real case, constants independent of $n$ do not generally exist, as shown in [2, Proposition 8.5].

In [5] it was shown that for any given operator ideal there is more than one ideal of polynomials compatible with it, for example the ideals of 2-dominated and multiple 2-summing 2-homogeneous polynomials are both compatible with the ideal of absolutely 2summing operators. Also, it was proved there that there exists at most one operator ideal compatible with a given polynomial ideal. On the other hand, not every polynomial ideal is compatible with the commonly associated operator ideal (e.g. the ideal absolutely 1summing polynomials is not compatible with the ideal of absolutely 1-summing operators [5, Example 1.15]).

It is natural to ask wether every polynomial ideal must have a (necessarily unique) compatible operator ideal or not. The following result answers this question affirmatively.

Theorem 2.3. Let $\mathfrak{A}_{n}$ be a Banach ideal of $n$-homogeneous polynomials. Then there exists a unique Banach ideal of operators $\mathfrak{A}$ compatible with $\mathfrak{A}_{n}$. Moreover, in the complex case the compatibility constants satisfy $1 \leq A, B \leq e$. 
The proof will be given in several steps and only for the complex case. For the real case, analogous versions of the next two lemmas can be obtained through the polarization formula. However, the resulting compatibility constants will depend on $n$.

Lemma 2.4. Let $\mathfrak{A}_{n}$ be a normed ideal of n-homogeneous polynomials and $P \in \mathfrak{A}_{n}(E, F)$. If $T, S \in \mathcal{L}(G, E)$, then the $n$-homogeneous polynomial $Q(\cdot)=\stackrel{\vee}{P}(T(\cdot), \cdots, T(\cdot), S(\cdot))$ belongs to $\mathfrak{A}_{n}(G, F)$. Moreover, $\|Q\|_{\mathfrak{A}_{n}(G, F)} \leq e\|T\|_{\mathcal{L}(G, E)}^{n-1}\|S\|_{\mathcal{L}(G, E)}\|P\|_{\mathfrak{A}_{n}(E, F)}$.

In particular, if $S \in \mathcal{L}(G, E), \gamma \in G^{\prime}$ and $a \in E$, then:

(a) $\gamma^{n-1}\left(P_{a^{n-1}} \circ S\right) \in \mathfrak{A}_{n}(G, F)$ with

$$
\left\|\gamma^{n-1}\left(P_{a^{n-1}} \circ S\right)\right\|_{\mathfrak{A}_{n}(G, F)} \leq e\|\gamma\|^{n-1}\|a\|^{n-1}\|P\|_{\mathfrak{A}_{n}(E, F)}\|S\|_{\mathcal{L}(G, E)} .
$$

(b) $\gamma\left(P_{a} \circ S\right) \in \mathfrak{A}_{n}(E, F)$ with

$$
\left\|\gamma\left(P_{a} \circ S\right)\right\|_{\mathfrak{A}_{n}(E, F)} \leq e\|\gamma\|\|a\|\|P\|_{\mathfrak{A}_{n}(E, F)}\|S\|^{n-1} .
$$

Proof. As in [5, Corollary 1.8], we can write $Q$ in the following useful way:

$$
Q(x)=\frac{1}{n^{2}} \frac{1}{(n-1)^{n-1}} \sum_{j=0}^{n-1} r^{j} P\left((n-1) r^{j} T(x)+S(x)\right),
$$

where $r$ is a primitive $n$th root of unity. Thus, defining, for each $0 \leq j \leq n-1$, the linear operator

$$
S_{j}(x)=(n-1) r^{j} T(x)+S(x),
$$

we have that

$$
Q=\frac{1}{n^{2}} \frac{1}{(n-1)^{n-1}} \sum_{j=0}^{n-1} r^{j}\left(P \circ S_{j}\right) .
$$

Therefore, $Q$ belongs to $\mathfrak{A}_{n}(G, F)$.

For the estimation of the norm, it is enough to consider the case $\|S\|=\|T\|=1$. Since $\left\|S_{j}\right\| \leq n$, for every $j=0, \ldots, n-1$, we obtain

$$
\|Q\|_{\mathfrak{A}_{n}(G, F)} \leq \frac{1}{n^{2}} \frac{1}{(n-1)^{n-1}} n\|P\|_{\mathfrak{A}_{n}(G, F)} n^{n}=\frac{n^{n-1}}{(n-1)^{n-1}}\|P\|_{\mathfrak{A}_{n}(G, F)} \leq e\|P\|_{\mathfrak{A}_{n}(G, F)} .
$$

For the particular cases, just note that $\gamma^{n-1}\left(P_{a^{n-1}} \circ S\right)(x)=\stackrel{\vee}{P}(\gamma(x) a, \cdots, \gamma(x) a, S(x))$, and $\gamma\left(P_{a} \circ S\right)(x)=\stackrel{\vee}{P}(\gamma(x) a, S(x), \cdots, S(x))$.

As a consequence of this lemma we obtain the following.

Lemma 2.5. Let $\mathfrak{A}_{n}$ be an ideal of $n$-homogeneous polynomials, let $T \in \mathcal{L}(E, F)$ and fix a nonzero $\gamma_{0} \in E^{\prime}$. Then $\gamma_{0}^{n-1} T \in \mathfrak{A}_{n}(E, F)$ if and only if $\gamma^{n-1} T \in \mathfrak{A}_{n}(E, F)$ for every $\gamma \in E^{\prime}$ 
EVERY BANACH IDEAL OF POLYNOMIALS IS COMPATIBLE WITH AN OPERATOR IDEAL 5

Proof. Assume that $\gamma_{0}^{n-1} T \in \mathfrak{A}_{n}(E, F)$ and pick $a \in E$ such that $\gamma_{0}(a) \neq 0$. By Lemma 2.4, $\gamma^{n-1}\left(\gamma_{0}^{n-1} T\right)_{a^{n-1}}$ belongs to $\mathfrak{A}_{n}(E, F)$. We have

$$
\gamma^{n-1}\left(\gamma_{0}^{n-1} T\right)_{a^{n-1}}(x)=\frac{\gamma(x)^{n-1}}{n}\left(\gamma_{0}(a)^{n-1} T(x)+(n-1) \gamma_{0}(a)^{n-2} \gamma_{0}(x) T(a)\right)
$$

Therefore

$$
\left(\gamma^{n-1} T\right)(\cdot)=\frac{n}{\gamma_{0}(a)^{n-1}}\left(\gamma^{n-1}(\cdot)\left(\gamma_{0}^{n-1} T\right)_{a^{n-1}}(\cdot)-\frac{n-1}{n} \gamma^{n-1}(\cdot) \gamma_{0}(\cdot) \gamma_{0}(a)^{n-2} T(a)\right),
$$

and then $\gamma^{n-1} T$ belongs to $\mathfrak{A}_{n}(E, F)$.

Now we can define, for a fixed polynomial ideal $\mathfrak{A}_{n}$, an operator ideal $\mathfrak{A}$, and a complete norm on it. This norm also has some interesting properties that we present in the following proposition.

Proposition 2.6. Let $\mathfrak{A}_{n}$ be an ideal of n-homogeneous polynomials. Define, for each pair of Banach spaces $E$ and $F$,

$$
\mathfrak{A}(E, F)=\left\{T \in \mathcal{L}(E, F): \gamma^{n-1} T \in \mathfrak{A}_{n}(E, F) \text { for all } \gamma \in E^{\prime}\right\},
$$

with $\|T \mid\|_{\mathfrak{A}(E, F)}=\sup _{\gamma \in S_{E^{\prime}}}\left\|\gamma^{n-1} T\right\|_{\mathfrak{A}_{n}(E, F)}$. Then

(a) $\mathfrak{A}$ is an ideal of operators and $\mathfrak{A}(E, F)=\left\{P_{a^{n-1}} \in \mathcal{L}(E, F) / P \in \mathfrak{A}_{n}(E, F), a \in E\right\}$.

(b) \|\|$\cdot \|_{\mathfrak{A}(E, F)}$ is a norm on $\mathfrak{A}(E, F)$ and satisfies

$$
\|T\|_{\mathfrak{A}(E, F)} \geq\|T\|_{\mathcal{L}(E, F)}, \quad \text { for every } T \in \mathfrak{A}(E, F) .
$$

Moreover, $\left(\mathfrak{A}(E, F),\|\cdot \cdot\|_{\mathfrak{A}(E, F)}\right)$ is a Banach space.

(c) $\|\mid S \circ T\|_{\mathfrak{A}\left(E, F_{1}\right)} \leq\|S\|_{\mathcal{L}\left(F, F_{1}\right)}\|T\|_{\|_{\mathfrak{A}(E, F)}}$ for all $S \in \mathcal{L}\left(F, F_{1}\right)$ and $T \in \mathfrak{A}(E, F)$.

(d) If $E_{0}$ is a subspace of $E$ with norm 1 inclusion $i: E_{0} \hookrightarrow E$, then

$$
\|T \circ i\|_{\mathfrak{A}\left(E_{0}, F\right)} \leq\|\mid\| T \|_{\mathfrak{A}(E, F)}, \quad \text { for all } T \in \mathfrak{A}(E, F) .
$$

Proof. (a) Clearly the sum and multiplication by scalars of members of $\mathfrak{A}$ are again in $\mathfrak{A}$. So, to prove that $\mathfrak{A}$ is an ideal of operators, we have to show that it behaves well with compositions.

Consider $T \in \mathfrak{A}(E, F), R \in \mathcal{L}\left(E_{1}, E\right)$ and $S \in \mathcal{L}\left(F, F_{1}\right)$. Let us prove that $S \circ T \circ R \in$ $\mathfrak{A}\left(E_{1}, F_{1}\right)$. Let $\gamma \in E^{\prime}$ such that $\gamma \circ R \neq 0$. Then $\gamma^{n-1} T \in \mathfrak{A}_{n}(E, F)$ and $\eta=\gamma \circ R \in E_{1}^{\prime}$. By Lemma 2.5, it suffices to show that $\eta^{n-1}(S \circ T \circ R) \in \mathfrak{A}_{n}\left(E_{1}, F_{1}\right)$. This follows from the equalities:

$$
\left(\eta^{n-1}(S \circ T \circ R)\right)(x)=\gamma^{n-1}(R(x)) S(T(R(x)))=\left(S \circ\left(\gamma^{n-1} T\right) \circ R\right)(x) .
$$

Therefore $\mathfrak{A}$ is an ideal of operators.

To prove the equivalent definition of $\mathfrak{A}$, suppose $T=P_{a^{n-1}}$ with $P \in \mathfrak{A}_{n}(E, F)$ and $a \in E$. Then by Lemma $2.4, \gamma^{n-1} T$ belongs to $\mathfrak{A}_{n}(E, F)$, for all $\gamma \in E^{\prime}$, and thus $T \in \mathfrak{A}(E, F)$. 
Conversely, if $T \in \mathfrak{A}(E, F)$ then $\gamma^{n-1} T \in \mathfrak{A}_{n}(E, F)$ for every $\gamma \in E^{\prime}$. Let $a \in E$ such that $\gamma(a)=1$, then $P:=n \gamma^{n-1} T-(n-1) T(a) \gamma^{n}$ is in $\mathfrak{A}_{n}(E, F)$ and $P_{a^{n-1}}=T$.

(b) It is straightforward to prove that we defined a norm.

Let $T \in \mathfrak{A}(E, F)$, take $x \in S_{E}$ such that $\|T(x)\|>\|T\|_{\mathcal{L}(E, F)}-\varepsilon$ and $\gamma \in S_{E^{\prime}}$ such that $|\gamma(x)|=1$. Then,

$$
\|T\|_{\mathfrak{A}(E, F)} \geq\left\|\gamma^{n-1} T\right\|_{\mathfrak{A}_{n}(E, F)} \geq\left\|\gamma^{n-1} T\right\|_{\mathcal{P}^{n}(E, F)} \geq\left\|\gamma(x)^{n-1} T(x)\right\|>\|T\|_{\mathcal{L}(E, F)}-\varepsilon .
$$

Since this is true for every $\varepsilon>0$, we have that $\|T\|_{\mathfrak{A}(E, F)} \geq\|T\|_{\mathcal{L}(E, F)}$.

Let us see that $\left(\mathfrak{A}(E, F),\|\| \cdot \|_{\mathfrak{A}(E, F)}\right)$ is complete. Suppose $\sum_{k \in \mathbb{N}}\left\|T_{k}\right\|_{\|_{\mathfrak{A}(E, F)}}$ is convergent. Then $\sum_{k \in \mathbb{N}}\left\|T_{k}\right\|_{\mathcal{L}(E, F)}$ is convergent. Therefore there exists $T \in \mathcal{L}(E, F)$ such that $\sum_{k} T_{k} \rightarrow T$ in $\mathcal{L}(E, F)$.

For each $\gamma \in S_{E^{\prime}}$, we know that $\gamma^{n-1} T_{k} \in \mathfrak{A}_{n}(E, F)$ and $\left\|\gamma^{n-1} T_{k}\right\|_{\mathfrak{A}_{n}(E, F)} \leq\|\| T_{k} \|_{\mathfrak{A}(E, F)}$. Thus, $\sum_{k} \gamma^{n-1} T_{k}$ converges in $\mathfrak{A}_{n}(E, F)$ and its limit has to be $\gamma^{n-1} T$. Therefore, $T$ belongs to $\mathfrak{A}(E, F)$. Moreover, since

$$
\sup _{\gamma \in S_{E^{\prime}}}\left\|\gamma^{n-1} \sum_{k \geq N} T_{k}\right\|_{\mathfrak{A}_{n}(E, F)} \leq \sup _{\gamma \in S_{E^{\prime}}} \sum_{k \geq N}\left\|\gamma^{n-1} T_{k}\right\|_{\mathfrak{A}_{n}(E, F)} \leq \sum_{k \geq N}\left\|T_{k}\right\|_{\mathfrak{A}(E, F)} \rightarrow 0
$$

as $N \rightarrow \infty$, we have that $\sum_{k} T_{k} \rightarrow T$ in $\left(\mathfrak{A}(E, F),\|\cdot \cdot\|_{\mathfrak{A}(E, F)}\right)$.

(c) For every $S \in \mathcal{L}\left(F, F_{1}\right)$ and $T \in \mathfrak{A}(E, F)$, we have:

$$
\begin{aligned}
\|S \circ T\|_{\mathfrak{A}\left(E, F_{1}\right)} & =\sup _{\gamma \in S_{E^{\prime}}}\left\|\gamma^{n-1} S \circ T\right\|_{\mathfrak{A}_{n}\left(E, F_{1}\right)}=\sup _{\gamma \in S_{E^{\prime}}}\left\|S \circ\left(\gamma^{n-1} T\right)\right\|_{\mathfrak{A}_{n}\left(E, F_{1}\right)} \\
& \leq\|S\|_{\mathcal{L}\left(F, F_{1}\right)} \sup _{\gamma \in S_{E^{\prime}}}\left\|\gamma^{n-1} T\right\|_{\mathfrak{A}_{n}(E, F)}=\|S\|_{\mathcal{L}\left(F, F_{1}\right)}\|T\|_{\mathfrak{A}(E, F)} .
\end{aligned}
$$

(d) Let $T \in \mathfrak{A}(E, F)$ and $\gamma \in E_{0}^{\prime}$. Consider $\tilde{\gamma} \in E^{\prime}$ a Hahn-Banach extension of $\gamma$ preserving its norm. Then

$\left\|\gamma^{n-1}(T \circ i)\right\|_{\mathfrak{A}_{n}\left(E_{0}, F\right)}=\left\|(\tilde{\gamma} \circ i)^{n-1}(T \circ i)\right\|_{\mathfrak{A}_{n}\left(E_{0}, F\right)}=\left\|\left(\tilde{\gamma}^{n-1} T\right) \circ i\right\|_{\mathfrak{A}_{n}\left(E_{0}, F\right)} \leq\left\|\tilde{\gamma}^{n-1} T\right\|_{\mathfrak{A}_{n}(E, F)}$.

Taking supremum we have that

$$
\|T \circ i\|_{\mathfrak{A}\left(E_{0}, F\right)} \leq\|\| T \|_{\mathfrak{A}(E, F)} .
$$

The following proposition shows that the norm defined on $\mathfrak{A}$ is "almost ideal", in the sense that satisfies the ideal condition up to a constant.

Proposition 2.7. The norm $\|\cdot \mid\|_{\mathfrak{A}}$ defined in Proposition 2.6 satisfies the "almost ideal" property: for Banach spaces $E$ and $F$, there exists a constant $c>0$ such that, for all Banach spaces $E_{1}, F_{1}$ and all operators $R \in \mathcal{L}\left(E_{1}, E\right), T \in \mathfrak{A}(E, F)$ and $S \in \mathcal{L}\left(F, F_{1}\right)$, it follows that

$$
\|S \circ T \circ R\|_{\mathfrak{A}\left(E_{1}, F_{1}\right)} \leq c\|S\|_{\mathcal{L}\left(F, F_{1}\right)}\|T\|_{\mathfrak{A}(E, F)}\|R\|_{\mathcal{L}\left(E_{1}, E\right)}
$$


EVERY BANACH IDEAL OF POLYNOMIALS IS COMPATIBLE WITH AN OPERATOR IDEAL 7

Proof. By Proposition $2.6(c)$, we have that

$$
\|S \circ T \circ R\|_{\mathfrak{A}\left(E_{1}, F_{1}\right)} \leq\|S\|_{\mathcal{L}\left(F, F_{1}\right)}\|T \circ R\|_{\mathfrak{A}\left(E_{1}, F\right)} .
$$

For a fixed Banach space $E_{1}$ and a fixed operator $R \in \mathcal{L}\left(E_{1}, E\right)$, consider

$$
\begin{array}{ccc}
\left(\mathfrak{A}(E, F),\|\mid \cdot\| \|_{\mathfrak{A}(E, F)}\right) & \rightarrow & \left(\mathfrak{A}\left(E_{1}, F\right),\|\| \cdot\|\|_{\mathfrak{A}\left(E_{1}, F\right)}\right) \\
T & \mapsto & T \circ R .
\end{array}
$$

An application of the Closed Graph Theorem gives the existence of a constant $c_{E_{1}, R}>0$ such that

$$
\|T \circ R\|_{\mathfrak{A}\left(E_{1}, F\right)} \leq c_{E_{1}, R}\|T\|_{\mathfrak{A}(E, F)} .
$$

If we apply again the Closed Graph Theorem for

$$
\begin{array}{ccc}
\mathcal{L}\left(E_{1}, E\right) & \rightarrow & \mathcal{L}\left(\mathfrak{A}(E, F), \mathfrak{A}\left(E_{1}, F\right)\right) \\
R & \mapsto & \theta_{R}(T)=T \circ R,
\end{array}
$$

we obtain a constant $c_{E_{1}}>0$ such that

$$
\|T \circ R\|_{\mathfrak{A}\left(E_{1}, F\right)} \leq c_{E_{1}}\|T\|_{\mathfrak{A}(E, F)}\|R\|_{\mathcal{L}\left(E_{1}, E\right)} .
$$

Now suppose that the result is not true. Then there are Banach spaces $E_{k}$, and $R_{k} \in$ $\mathcal{L}\left(E_{k}, E\right),\left\|R_{k}\right\|_{\mathcal{L}\left(E_{k}, E\right)}=1$, for all $k \in \mathbb{N}$, such that

$$
\left\|T \circ R_{k}\right\|_{\mathfrak{A}\left(E_{k}, F\right)}>k .
$$

Let $E_{0}=\bigoplus_{k \in \mathbb{N}} E_{k}$, and $\tilde{R}_{k} \in \mathcal{L}\left(E_{0}, E\right), \tilde{R}_{k}=R_{k} \circ \pi_{k}$, where $\pi_{k}: E_{0} \rightarrow E_{k}$ is the (norm one) projection. Denote by $i_{k}: E_{k} \hookrightarrow E_{0}$ the (norm one) inclusion. So we have

$$
\begin{aligned}
k & <\left\|T \circ R_{k}\right\|_{\mathfrak{A}\left(E_{k}, F\right)}=\left\|T \circ R_{k} \circ \pi_{k} \circ i_{k}\right\|_{\mathfrak{A}\left(E_{k}, F\right)} \\
& =\left\|T \circ \tilde{R}_{k} \circ i_{k}\right\|_{\mathfrak{A}\left(E_{k}, F\right)} \leq\left\|T \circ \tilde{R}_{k}\right\|_{\mathfrak{A}\left(E_{0}, F\right)},
\end{aligned}
$$

the last inequality following from Proposition 2.6(d). Also, by (2),

$$
\left\|T \circ \tilde{R}_{k}\right\|\left\|_{\mathfrak{A}\left(E_{0}, F\right)} \leq c_{E_{0}}\right\| T\left\|_{\mathfrak{A}(E, F)}\right\| \tilde{R}_{k}\left\|_{\mathcal{L}\left(E_{0}, E\right)} \leq c_{E_{0}}\right\| T \|_{\mathfrak{A}(E, F)},
$$

which leads to a contradiction.

Now we present a result that shows how to convert an "almost ideal" norm into an ideal norm.

Proposition 2.8. Let $\mathfrak{A}$ be an operator ideal with a norm $\|\cdot\| \|_{\mathfrak{A}}$ that satisfies the "almost ideal" property. Then we can define an equivalent norm $\|\cdot\|_{\mathfrak{A}}$ which is an ideal norm on $\mathfrak{A}$. 
Proof. We first define a norm $\|\cdot\|_{\mathfrak{A}}^{\prime}$ in the following way. For $T \in \mathfrak{A}(E, F)$, let $\|T\|_{\mathfrak{A}(E, F)}^{\prime}=\sup \left\{\|S \circ T \circ R\|_{\mathfrak{A}\left(E_{1}, F_{1}\right)}: E_{1}, F_{1}\right.$ Banach spaces, $\left.\|S\|_{\mathcal{L}\left(F, F_{1}\right)}=\|R\|_{\mathcal{L}\left(E_{1}, E\right)}=1\right\}$. It is easy to see that $\|\cdot\|_{\mathfrak{A}}^{\prime}$ is a norm on $\mathfrak{A}$ equivalent to \|\|$\cdot\|\|_{\mathfrak{A}}$. Also, it is clear that it satisfies the ideal property:

$$
\|S \circ T \circ R\|_{\mathfrak{A}\left(E_{1}, F_{1}\right)}^{\prime} \leq\|S\|_{\mathcal{L}\left(F, F_{1}\right)}\|T\|_{\mathfrak{A}(E, F)}^{\prime}\|R\|_{\mathcal{L}\left(E_{1}, E\right)}
$$

Last, if $\kappa=\left\|i d_{\mathbb{C}}\right\|_{\mathfrak{A}(\mathbb{C}, \mathbb{C})}^{\prime}$ then the norm $\|\cdot\|_{\mathfrak{A}}$ defined by

$$
\|T\|_{\mathfrak{A}(E, F)}=\frac{1}{\kappa}\|T\|_{\mathfrak{A}(E, F)}^{\prime}
$$

is an ideal norm equivalent to $\||\cdot|\|_{\mathfrak{A}}$.

Remark 2.9. We want to apply the previous proposition to an operator ideal $\left(\mathfrak{A},\|\cdot \cdot\|_{\mathfrak{A}}\right)$ built from a polynomial ideal $\mathfrak{A}_{n}$ as in (1). By Proposition 2.6 (c), we can simplify the definition of $\|\cdot\|_{\mathfrak{A}}^{\prime}$ for this case as follows:

$$
\|T\|_{\mathfrak{A}(E, F)}^{\prime}=\sup \left\{\|T \circ R\|_{\mathfrak{A}\left(E_{1}, F\right)}: E_{1} \text { Banach space, }\|R\|_{\mathcal{L}\left(E_{1}, E\right)}=1\right\} \text {. }
$$

Then considering

$$
\|T\|_{\mathfrak{A}(E, F)}=\frac{\|T\|_{\mathfrak{A}(E, F)}^{\prime}}{\left\|i d_{\mathbb{C}}\right\|_{\mathfrak{A}(\mathbb{C}, \mathbb{C})}^{\prime}}
$$

we obtain an ideal norm on $\mathfrak{A}$ equivalent to $\||\cdot|\|_{\mathfrak{A}}$. Moreover,

$$
\begin{aligned}
\kappa & =\|z \mapsto z\|_{\mathfrak{A}(\mathbb{C}, \mathbb{C})}^{\prime}=\sup \left\{\|(z \mapsto z) \circ \varphi\|_{\mathfrak{A}\left(E_{1}, \mathbb{C}\right)}: E_{1} \text { Banach space, } \varphi \in S_{E_{1}^{\prime}}\right\} \\
& =\sup \left\{\|\varphi\|_{\mathfrak{A}\left(E_{1}, \mathbb{C}\right)}: E_{1} \text { Banach space, } \varphi \in S_{E_{1}^{\prime}}\right\} \\
& =\sup \left\{\left\|\gamma^{n-1} \varphi\right\|_{\mathfrak{A}_{n}\left(E_{1}, \mathbb{C}\right)}: E_{1} \text { Banach space, } \varphi, \gamma \in S_{E_{1}^{\prime}}\right\} .
\end{aligned}
$$

Thus by [5, Corollary 1.8], we have that $1 \leq \kappa \leq e$.

We can now prove the existence, for any polynomial ideal, of a compatible operator ideal:

Proof. (of Theorem 2.3) Consider the normed ideal $\left(\mathfrak{A},\|\cdot\|_{\mathfrak{A}}\right)$, with

$$
\mathfrak{A}(E, F)=\left\{T \in \mathcal{L}(E, F) / \gamma^{n-1} T \in \mathfrak{A}_{n}(E, F) \text { for all } \gamma \in E^{\prime}\right\}
$$

and $\|\cdot\|_{\mathfrak{A}}$ given by Remark $2.9(\mathrm{ii})$. By the equivalence with \|\|$\cdot\|\|_{\mathfrak{A}}$ and Proposition $2.6(b)$, for each $E$ and $F$ Banach, $\left(\mathfrak{A}(E, F),\|\cdot\|_{\mathfrak{A}(E, F)}\right)$ is a Banach space.

Let us check that $\mathfrak{A}_{n}$ is compatible with $\mathfrak{A}$.

It is clear, by definition, that if $T \in \mathfrak{A}(E, F)$ and $\gamma \in E^{\prime}$ then $\gamma^{n-1} T \in \mathfrak{A}_{n}(E, F)$. On the other hand take $P \in \mathfrak{A}_{n}(E, F)$ and $a \in E$. By Proposition $2.6(a), P_{a^{n-1}}$ belongs to 
EVERY BANACH IDEAL OF POLYNOMIALS IS COMPATIBLE WITH AN OPERATOR IDEAL 9 $\mathfrak{A}(E, F)$. By Remark 2.2 we conclude that $\mathfrak{A}_{n}$ is compatible with $\mathfrak{A}$. We can moreover estimate the constants of compatibility. For the first one, by Lemma $2.4(a)$,

$$
\left\|P_{a^{n-1}}\right\|_{\mathfrak{A}(E, F)}=\frac{1}{\kappa} \sup _{\substack{E_{1} \text { Banach } \\ R \in S_{\mathcal{L}\left(E_{1}, E\right)}}} \sup _{\|\gamma\|=1}\left\|\gamma^{n-1}\left(P_{a^{n-1}} \circ R\right)\right\|_{\mathfrak{A}_{n}\left(E_{1}, F\right)} \leq \frac{e}{\kappa}\|a\|^{n-1}\|P\|_{\mathfrak{A}_{n}(E, F)} .
$$

For the other constant we have,

$$
\left\|\gamma^{n-1} T\right\|_{\mathfrak{A}_{n}(E, F)}=\|\gamma\|^{n-1}\left\|\frac{\gamma^{n-1}}{\|\gamma\|^{n-1}} T\right\|_{\mathfrak{A}_{n}(E, F)} \leq\|\gamma\|^{n-1}\|T\|_{\mathfrak{A}(E, F)} \leq \kappa\|\gamma\|^{n-1}\|T\|_{\mathfrak{A}(E, F)} .
$$

The fact that $\mathfrak{A}$ is the only ideal of operators compatible with $\mathfrak{A}_{n}$ follows from [5, Proposition 1.6].

We have proved that every polynomial Banach ideal is compatible with a unique Banach operator ideal. On the other hand, Example 1.15 in [5] shows that the ideal of absolutely 1summing polynomials is not compatible with the ideal of absolutely 1-summing operators. Then the question that comes up is which ideal of linear operators is compatible with the ideal of absolutely 1-summing polynomials.

As the following proposition shows, the unique compatible operator ideal may be far from "natural". Note, however, that this unnatural compatibility has some interesting consequences (see Corollary 2.11 and the comments after Example 3.2).

Proposition 2.10. The ideal $\Pi_{p}^{n}$ of absolutely p-summing n-homogeneous polynomials is compatible with $\mathcal{L}$, the ideal of continuous linear operators, with constants $A=e$ and $B=1$.

Proof. Obviously, for $P \in \Pi_{p}^{n}(E, F)$ and $a \in E$, the operator $P_{a^{n-1}}$ belongs to $\mathcal{L}(E, F)$ and

$$
\left\|P_{a^{n-1}}\right\|_{\mathcal{L}(E, F)} \leq e\|P\|_{\mathcal{P}^{n}(E, F)}\|a\|^{n-1} \leq e\|P\|_{\Pi_{p}^{n}(E, F)}\|a\|^{n-1} .
$$

For the other condition, let $T \in \mathcal{L}(E, F)$ and $\gamma \in E^{\prime}$. Then, for all $x_{1}, \ldots, x_{m} \in E$,

$$
\begin{aligned}
\left(\sum_{j=1}^{m}\left\|\left(\gamma^{n-1} T\right)\left(x_{j}\right)\right\|^{p}\right)^{\frac{1}{p}} & \leq\|\gamma\|\left(\sum_{j=1}^{m}\left(\frac{\left|\gamma\left(x_{j}\right)\right|}{\|\gamma\|}\|\gamma\|^{n-2}\|T\|\left\|x_{j}\right\|^{n-1}\right)^{p}\right)^{\frac{1}{p}} \\
& \leq\|\gamma\|^{n-1}\|T\|\left(\sum_{j=1}^{m}\left(\frac{\left|\gamma\left(x_{j}\right)\right|}{\|\gamma\|}\right)^{p}\right)^{\frac{1}{p}}\left(\max _{1 \leq j \leq m}\left\|x_{j}\right\|\right)^{n-1} \\
& \leq\|\gamma\|^{n-1}\|T\| \sup _{x^{\prime} \in B_{E^{\prime}}}\left(\sum_{j=1}^{m}\left|x^{\prime}\left(x_{j}\right)\right|^{p}\right)^{\frac{n}{p}}=\|\gamma\|^{n-1}\|T\| \omega_{p}\left(\left(x_{j}\right)_{j=1}^{m}\right)^{n} .
\end{aligned}
$$

Thus, $\gamma^{n-1} T$ is absolutely $p$-summing and

$$
\left\|\gamma^{n-1} T\right\|_{\Pi_{p}^{n}(E, F)} \leq\|T\|_{\mathcal{L}(E, F)}\|\gamma\|^{n-1} .
$$


It is well known that every absolutely summing operator is weakly compact (see for example [7, Theorem 2.17]). In [1] it was shown that not every dominated polynomial is weakly compact by exhibiting an example of a polynomial from $\ell_{1}$ to $\ell_{1}$. We now show how the concept of compatible ideals can be easily applied to prove that not every absolutely $p$-summing homogeneous polynomial is weakly compact.

Corollary 2.11. E is reflexive if and only if, for some $n \geq 2$, every absolutely $p$-summing $n$-homogeneous polynomial from $E$ to $E$ is weakly compact.

Proof. It easy to prove that the ideal of weakly compact homogeneous polynomials, $\mathcal{P}_{W K}^{n}$, is compatible with the ideal of weakly compact operators, $\mathcal{L}_{W K}$. Suppose that $\Pi_{p}^{n}(E, E) \subset$ $\mathcal{P}_{W K}^{n}(E, E)$. Then, by [5, Proposition 1.6], we have that $\mathcal{L}(E, E)=\mathcal{L}_{W K}(E, E)$ and thus $E$ must be reflexive.

Conversely, if $E$ is reflexive, every homogeneous polynomial from $E$ to $E$ is weakly compact.

The non-trivial implication of the previous corollary can be also established as follows: the polynomial $P(x)=\gamma(x)^{n-1} x$, where $\gamma$ is a nonzero functional, is absolutely $p$-summing by Proposition 2.10, and hence weakly compact by assumption. Now the reflexivity of $E$ follows from item $(c)$ of the Proposition in page 461 of [1].

Analogously we can prove that if every absolutely $p$-summing $n$-homogeneous polynomial from $E$ to $F$ is weakly compact (for some $n \geq 2$ ), then every linear operator from $E$ to $F$ is weakly compact.

\section{COHERENT SEQUENCES OF POLYNOMIAL IDEALS}

In [5] we also defined coherent sequences of polynomial ideals. In view of [2, Proposition 8.5], coherence is too restrictive for the real case and, as a consequence, we will restrict ourselves to the complex case. All Banach spaces considered in this Section will be complex.

Definition 3.1. Consider the sequence $\left\{\mathfrak{A}_{k}\right\}_{k=1}^{N}$, where for each $k, \mathfrak{A}_{k}$ is an ideal of $k$ homogeneous polynomials and $N$ is eventually infinite. We say that $\left\{\mathfrak{A}_{k}\right\}_{k}$ is a coherent sequence of polynomial ideals if there exist positive constants $C$ and $D$ such that for all Banach spaces $E$ and $F$, the following conditions hold for $k=1, \ldots, N-1$ :

(i) For each $P \in \mathfrak{A}_{k+1}(E, F)$ and $a \in E, P_{a}$ belongs to $\mathfrak{A}_{k}(E ; F)$ and

$$
\left\|P_{a}\right\|_{\mathfrak{A}_{k}(E, F)} \leq C\|P\|_{\mathfrak{A}_{k+1}(E, F)}\|a\| .
$$

(ii) For each $P \in \mathfrak{A}_{k}(E, F)$ and $\gamma \in E^{\prime}, \gamma P$ belongs to $\mathfrak{A}_{k+1}(E, F)$ and

$$
\|\gamma P\|_{\mathfrak{A}_{k+1}(E, F)} \leq D\|\gamma\|\|P\|_{\mathfrak{A}_{k}(E, F)} .
$$


Note that property $(i i)$ in Definition 3.1 implies that the polynomial ideal is closed for scalar multiplication (in the sense of [3]), and property $(i)$ in Definition 3.1 is what in [2] is called "polynomial property $(B)$ ". Also, coherent sequences are always global holomorphy types.

It is shown in [5] that, given an operator ideal $\mathfrak{A}$, there are many coherent sequences $\left\{\mathfrak{A}_{k}\right\}_{k}$ such that $\mathfrak{A}_{1}=\mathfrak{A}$. On the other hand, for a given ideal of $n$-homogeneous polynomials $\mathfrak{A}_{n}$ there can be at most one coherent sequence $\left\{\mathfrak{A}_{1}, \mathfrak{A}_{2}, \ldots, \mathfrak{A}_{n}\right\}$. In other words, all coherent sequences with the same $n$-homogeneous ideal must have the same $k$-homogeneous ideals for $1 \leq k \leq n$. As in the case of compatibility, not all the usual polynomial extensions of an operator ideal form a coherent sequence. Indeed, an argument similar to the proof of Proposition 2.10 proves:

Example 3.2. The sequence $\left\{\mathcal{L}, \mathcal{P}^{2}, \ldots, \mathcal{P}^{n-1}, \Pi_{p}^{n}\right\}$ is coherent with constants $C=e$ and $D=1$.

From this example and [5, Proposition 1.6] we can prove that if every absolutely $p$ summing $n$-homogeneous polynomial from $E$ to $F$ is weakly compact, then every $k$ homogeneous polynomial from $E$ to $F$ is weakly compact, for each $k \leq n-1$.

Moreover, since $\left\{\mathcal{L}, \mathcal{P}^{2}, \ldots, \mathcal{P}^{n-1}, \Pi_{p}^{n}\right\}$ is a coherent sequence, it may be shown, as in Proposition $2.6(a)$, that for all Banach spaces $E, F$,

$$
\mathcal{P}^{n-1}(E, F)=\left\{P_{a}: P \in \Pi_{p}^{n}(E, F), a \in E\right\} .
$$

Therefore we can conclude that if $\mathcal{P}^{n}(E, F) \neq \Pi_{p}^{n}(E, F)$ for some $n \geq 1$, then the sequence $\left\{\Pi_{p}^{k}(E, F)\right\}_{k \in \mathbb{N}}$ is not a holomorphy type. This improves [2, Example 2.2].

For $\mathfrak{A}_{n}$ a Banach ideal of $n$-homogeneous polynomials, we can define for each Banach spaces $E, F$,

$$
\mathfrak{A}_{n-1}(E, F)=\left\{P \in \mathcal{P}^{n-1}(E, F): \gamma P \in \mathfrak{A}_{n}(E, F) \text { for all } \gamma \in E^{\prime}\right\},
$$

with $\|\mid\| P\left\|_{\mathfrak{A}_{n-1}(E, F)}=\sup _{\gamma \in S_{E^{\prime}}}\right\| \gamma P \|_{\mathfrak{A}_{n}(E, F)}$. With some modifications in the results from the previous section (see [12] for details) it may be proven that $\mathfrak{A}_{n-1}$ is an ideal of $(n-1)$ homogenous polynomials and that $\|\cdot \cdot\|_{\mathfrak{A}_{n-1}}$ is an almost ideal norm, which can be modified to an equivalent ideal norm $\|\cdot\|_{\mathfrak{A}_{n}(E, F)}$. We can proceed analogously to define $\mathfrak{A}_{n-2}$, and then $\mathfrak{A}_{n-3}, \ldots, \mathfrak{A}_{1}$. As a result, we have shown how to construct a (necessarily unique) sequence of polynomial ideals $\mathfrak{A}_{1}, \ldots, \mathfrak{A}_{n-1}$ such that $\left\{\mathfrak{A}_{1}, \ldots, \mathfrak{A}_{n-1}, \mathfrak{A}_{n}\right\}$ is a coherent sequence. Also, the polynomial ideals $\mathfrak{A}_{1}, \ldots, \mathfrak{A}_{n-1}$ can be normed to obtain constants of coherence $1 \leq C, D \leq e$.

We can also give a whole sequence $\left\{\mathfrak{A}_{k}\right\}_{k=1}^{\infty}$ of polynomial ideals which form a coherent sequence. In this case the ideals $\mathfrak{A}_{k}, k \geq n+1$ are not uniquely determined by $\mathfrak{A}_{n}$. For example, we may define for each Banach spaces $E, F, k \geq 1$,

$$
\mathfrak{A}_{n+k}(E, F)=\left\{P \in \mathcal{P}^{n+k}(E, F): P_{a^{k}} \text { belongs to } \mathfrak{A}_{n}(E, F) \text { for every } a \in E\right\},
$$


and $\|P\|_{\mathfrak{A}_{n+k}(E, F)}=\sup \left\{\left\|P_{a^{k}}\right\|_{\mathfrak{A}_{n}(E, F)}:\|a\|_{E}=1\right\}$. Then it is easy to see that $\mathfrak{A}_{n+k}$ is an ideal of $(n+k)$-homogeneous polynomial and that $\|\cdot\|_{\mathfrak{A}_{n+k}}$ is an ideal norm. Moreover, a simple modification of the proof of Proposition 2.4 a) in [5] shows that if $P \in \mathfrak{A}_{n+k}(E, F)$ and $a \in E$ then $P_{a}$ belongs to $\mathfrak{A}_{n+k-1}(E, F)$ and $\left\|P_{a}\right\|_{\mathfrak{A}_{n+k-1}(E, F)} \leq e\|a\|\|P\|_{\mathfrak{A}_{n+k}(E, F)}$. Therefore we have the following.

Theorem 3.3. Let $\mathfrak{A}_{n}$ be a Banach ideal of n-homogeneous polynomials. Then there exist polynomial ideals $\mathfrak{A}_{1}, \ldots, \mathfrak{A}_{n-1}, \mathfrak{A}_{n+1}, \ldots$ such that $\left\{\mathfrak{A}_{k}\right\}_{k=1}^{\infty}$ is a coherent sequence with constants $1 \leq C, D \leq e$. The polynomial ideals $\mathfrak{A}_{1}, \ldots, \mathfrak{A}_{n-1}$ are uniquely determined by $\mathfrak{A}_{n}$.

The sequence $\left\{\mathfrak{A}_{k}\right\}_{k=1}^{\infty}$ constructed is actually the largest sequence of ideals coherent with $\mathfrak{A}_{n}$. That is, if $\left\{\mathfrak{B}_{k}\right\}_{k=1}^{\infty}$ is a coherent sequence such that $\mathfrak{B}_{n}=\mathfrak{A}_{n}$, then $\mathfrak{B}_{k}(E, F) \subset$ $\mathfrak{A}_{k}(E, F)$ for all $E, F, k \in \mathbb{N}$ (equality holds for $k<n$ ). It is also possible to define the smallest coherent sequence associated to $\mathfrak{A}_{n}$, see [5, Section 2] for a related construction.

It is clear that we can deduce the existence of compatible operator ideal for every polynomial ideal from Theorem 3.3. However, is should be noted that the bounds for the compatibility constants obtained with this theorem would be $e^{n}$, while in Theorem 2.3 we have $e$ as a bound.

\section{ACKNOWLEDGEMENTS}

We would like to thank the anonymous referee for his/her valuable comments and suggestions.

\section{REFERENCES}

[1] Geraldo Botelho, Weakly compact and absolutely summing polynomials, J. Math. Anal. Appl. 265 (2002), no. 2, 458-462.

[2] Geraldo Botelho, Hans A. Braunss, H. Junek, and D. Pellegrino, Holomorphy types and ideals of multilinear mappings, Stud. Math. 177 (2006), no. 1, 43-65.

[3] Geraldo Botelho, and Daniel Pellegrino, Two new properties of ideals of polynomials and applications, Indag. Math. (N.S.) 16 (2005), no. 2, 157-169.

[4] Hans A. Braunss, Ideale multilinearer Abbildungen und Räume holomorpher Funktionen, Ph.D. Thesis, Postdam, 1984.

[5] Daniel Carando, Verónica Dimant, and Santiago Muro, Coherent sequences of polynomial ideals on Banach spaces, Math. Nachr. 282 (2009), no. 8, 1111-1133.

[6] _ Holomorphic Functions and polynomial ideals on Banach spaces, Collect. Math. (to appear).

[7] Joe Diestel, Hans Jarchow, and Andrew Tonge, Absolutely summing operators, Cambridge Studies in Advanced Mathematics, vol. 43, Cambridge University Press, Cambridge, 1995.

[8] Dineen, Seán. Holomorphy types on a Banach space. Studia Math. 39 (1971), 241-288.

[9] Klaus Floret, Minimal ideals of n-homogeneous polynomials on Banach spaces, Result. Math. 39 (2001), no. 3-4, 201-217. 
EVERY BANACH IDEAL OF POLYNOMIALS IS COMPATIBLE WITH AN OPERATOR IDEAL 13

[10] _ On ideals of n-homogeneous polynomials on Banach spaces, Topological algebras with applications to differential geometry and mathematical physics (Athens, 1999), Univ. Athens, Athens, 2002, 19-38.

[11] Klaus Floret and Stephan Hunfeld, Ultrastability of ideals of homogeneous polynomials and multilinear mappings on Banach spaces, Proc. Am. Math. Soc. 130 (2002), no. 5, 1425-1435.

[12] Santiago Muro, Funciones holomorfas de tipo acotado e ideales de polinomios homogéneos en espacios de Banach, Ph.D. thesis, Univ. de Buenos Aires, 2010.

[13] Leopoldo Nachbin, Topology on spaces of holomorphic mappings, Ergebnisse der Mathematik und ihrer Grenzgebiete, Band 47, Springer-Verlag New York Inc., New York, 1969.

Departamento de Matemática - Pab i, Facultad de Cs. Exactas y Naturales, UniversiDad de Buenos Aires, (1428) Buenos Aires, Argentina and COniCET.

E-mail address: dcarando@dm.uba.ar

Departamento de Matemática, Universidad de San Andrés, Vito Dumas 284, (B1644BID) Victoria, Buenos Aires, Argentina and COniCET.

E-mail address: vero@udesa.edu.ar

Departamento de Matemática - Pab i, Facultad de Cs. Exactas y Naturales, Universidad de Buenos Aires, (1428) Buenos Aires, Argentina and COniCET.

E-mail address: smuro@dm.uba.ar 